\title{
Surgical Recommendations in Ehlers-Danlos Syndrome(s) Need Patient Classification: The Example of Ehlers-Danlos Syndrome Hypermobility Type (a.k.a. Joint Hypermobility Syndrome)
}

\author{
Marco Castori \\ Division of Medical Genetics, Department of Molecular Medicine, Sapienza University, San Camillo-Forlanini Hospital, \\ Rome, Italy
}

Dear Sir,

I read with great interest the work by Burcharth and Rosenberg [1] (Herlev, Denmark) on surgical aspects of Ehlers-Danlos syndrome(s) (EDSs). In this work, the authors review 53 previously published papers, summarize indications and complications by anatomic structure, and define some practical recommendations for improving outcomes of skin closure after surgical incisions. Their work is definitely of invaluable support in the daily practice of many professionals involved in the management of hereditary connective tissue disorders.

However, the extreme clinical variability and genetic heterogeneity of EDSs represent a major limit to such generalizations. In fact, as stated by the authors themselves in the first paragraph of their paper, EDS actually groups together an increasing number of genetic disorders sharing various degrees of generalized joint hypermobility and some cutaneous and soft tissue features, but with differences in the spectrum of ancillary findings and the molecular basis. Such discordance impacts prognosis which diverges significantly among clinical subtypes. Accordingly, it has not been emphasized enough that most papers included in the work by Burcharth and Rosenberg report patients with the vascular type of EDS (or EDS type IV) or who show less characterized phenotypes and/or are described before the publication of the present classification [2]. The degree of soft tissue, skin and vascular fragility varies among the three most common subtypes of EDS, namely classic, hypermobility (EDS-HT) and vascular types. Therefore, it is reasonable that surgical risks are not the same among these variants.
In particular, EDS-HT is probably the most common EDS subtype [3] and likely represents one and the same disorder as the joint hypermobility syndrome (JHS) [4]. Therefore, it is reasonable that JHS/EDS-HT is the EDS form most frequently encountered in many surgery subspecialties, except perhaps in vascular and chest clinics. Not many data have been published concerning surgical aspects of JHS/EDS-HT. In contrast to vascular EDS in which surgery is typically associated with high risks, daily practice indicates that preventive contraindication to surgery is not a feature of JHS/EDS-HT. Nevertheless, a series of recently accumulated evidence and anecdotal reports depicts an extraordinarily complex pathophysiology of JHS/EDS-HT, which extends much beyond the involvement of the integumentary and musculoskeletal systems. Accordingly, many factors may influence the outcome of surgical and anesthetic procedures and should be considered during treatment planning. Table 1 summarizes available data and the ensuing recommendations in JHS/EDS-HT.

I hope that this additional information will help to offer a more tailored surgical approach for patients affected by the JHS/EDSHT subtype. It is expected that future studies will improve our knowledge on the widespread consequences of JHS/EDS-HT, as well as other major EDS subtypes, in order to select more efficient and personalized management interventions.

\section{Disclosure Statement}

The author has no conflict of interest to disclose.

\section{KARGER}

Fax +4161306 1234 E-Mail karger@karger.com www.karger.com
(C) 2012 S. Karger AG, Basel

$0253-4886 / 12 / 0296-0453 \$ 38.00 / 0$

Accessible online at:

www.karger.com/dsu
Marco Castori, MD

Division of Medical Genetics, Department of Molecular Medicine, Sapienza University, San Camillo-Forlanini Hospital Circonvallazione Gianicolense, 87, IT-00152 Rome (Italy)

E-Mail mcastori@scamilloforlanini.rm.it 
Table 1. Surgical and anesthetic recommendations in the JHS/EDS-HT

Surgical procedure

(1) Orthopedic surgery is paradoxically associated with pain worsening in JHS/EDS-HT; anecdotal observations suggest a low success rate for abdominal surgery in functional disorders

(2) Although soft tissue fragility is not severe in JHS/EDS-HT, delayed wound healing with consequent suture widening, suture dehiscence and postsurgical hernias are possible complications

(3) Minor bleeding disorders are common in JHS/EDS-HT

(4) Episiotomy is associated with an increased risk for pelvic prolapses in JHS/EDS-HT women

Anesthetic procedure

(5) Dysautonomia is a major feature in JHS/EDS-HT and may need special anesthetic considerations

(6) JHS/EDS-HT patients often display resistance to intradermal lidocaine infiltrations and topical EMLA cream

(7) Epi/peridural anesthesia may be hampered by severe spondylosis and/or scoliosis, and could be complicate by intraspinal hypotension due to increased meningeal weakness in JHS/EDS-HT

(8) Temporomandibular joint dysfunction and occipitoatlantoaxial instability may be more common in JHS/EDS-HT
5 Consider more conservative treatments as an alternative to non-lifethreatening operations

1,6 (a) Perform skin closure in two layers (cutaneous and subcutaneous) without excessive tension

(b) Use generous sutures, deep stitches and steri-strips as reinforcement devices

(c) Leave sutures twice as long as normally recommended

Consider preoperative prophylaxis with desmopressin (1-deamino-

8 -D-arginine vasopressin), especially in patients with a positive history for mucosal bleeding (nose, gingivae, bowel, bladder, etc.) and/or easy bruising

$8 \quad$ Consider cesarean section as first-choice delivery procedure
Postsurgery recovery

(9) Muscle deconditioning due to inactivity rapidly worsens chronic pain and fatigue in JHS/EDS-HT (a) Consider to carry out appropriate investigations (e.g. tilt test) before any intervention in order to properly plan the anesthetic procedure, especially in patients with cardiovascular symptoms

(b) In case of confirmed dysautonomia, consider prophylactic early fluid loading and phenylephrine infusion

10, 11 Consider alternative anesthetic procedures or double the anesthetic dose

None $^{1} \quad$ Favor total anesthesia in case of major surgery

13, 14 Perform intubation with care and consider the use of pediatric devices also in adults

${ }^{1}$ Reports specifically describing such likely complications are lacking. However, mild scoliosis and premature spondylosis are commonly encountered in the JHS/EDS-HT clinic, while some preliminary studies indicate that generalized joint hypermobility is associated with orthostatic headache [12].

\section{References}

1 Burcharth J, Rosenberg J: Gastrointestinal surgery and related complications in patients with Ehlers-Danlos syndrome: a systematic review. Dig Surg 2012;29:349-357.

2 Beighton P, De Paepe A, Steinmann B, Tsipouras P, Wenstrup RJ: Ehlers-Danlos syndromes: revised nosology, Villefranche, 1997. Ehlers-Danlos National Foundation (USA) and Ehlers-Danlos Support Group (UK). Am J Med Genet 1998;77:31-37.

3 Hakim AJ, Sahota A: Joint hypermobility and skin elasticity: the hereditary disorders of connective tissue. Clin Dermatol 2006;24: 521-533.
4 Tinkle BT, Bird HA, Grahame R, Lavallee M, Levy HP, Sillence D: The lack of clinical distinction between the hypermobility type of Ehlers-Danlos syndrome and the joint hypermobility syndrome (a.k.a. hypermobility syndrome). Am J Med Genet A 2009; 149A:2368-2370.

5 Voermans NC, Knoop H, Bleijenberg G, van Engelen BG: Pain in Ehlers-Danlos syndrome is common, severe, and associated with functional impairment. J Pain Symptom Manage 2010;40:370-378.

6 Castori M: Ehlers-Danlos syndrome, hypermobility type (a.k.a. joint hypermobility syndrome): an updated critique. G Ital Dermatol Venereol, in press.
7 Jackson SC, Odiaman L, Card RT, van der Bom JG, Poon MC: Suspected collagen disorders in the bleeding disorder clinic: a casecontrol study. Haemophilia 2012, E-pub ahead of print.

8 Castori M, Morlino S, Dordoni C, Celletti C, Camerota F, Ritelli M, Morrone A, Venturini M, Grammatico P, Colombi M: Gynecologic and obstetric implications of the joint hypermobility syndrome (a.k.a. Ehlers-Danlos syndrome hypermobility type) in 82 Italian patients. Am J Med Genet A 2012;158A:21762182 . 
9 Mathias CJ, Low DA, Iodice V, Owens AP, Kirbis M, Grahame R: Postural tachycardia syndrome - current experience and concepts. Nat Rev Neurol 2011;8:22-34.

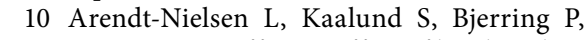
Høgsaa B: Insufficient effect of local analgesics in Ehlers Danlos type III patients (connective tissue disorder). Acta Anaesthesiol Scand 1990;34:358-361.

11 Hakim AJ, Grahame R, Norris P, Hopper C: Local anaesthetic failure in joint hypermobility syndrome. J R Soc Med 2005;98:84-85.
2 Schievink WI, Gordon OK, Tourje J: Connective tissue disorders with spontaneous spinal cerebrospinal fluid leaks and intracranial hypotension: a prospective study. Neurosurgery 2004;54:65-70.

13 De Coster PJ, Van den Berghe LI, Martens LC: Generalized joint hypermobility and temporomandibular disorders: inherited connective tissue disease as a model with maximum expression. J Orofac Pain 2005; 19:47-57.
14 Milhorat TH, Bolognese PA, Nishikawa M, McDonnell NB, Francomano CA: Syndrome of occipitoatlantoaxial hypermobility, cranial settling, and Chiari malformation type I in patients with hereditary disorders of connective tissue. J Neurosurg Spine 2007;7: 601-609.

15 Castori M, Morlino S, Celletti C, Celli M, Morrone A, Colombi M, Camerota F, Grammatico P: Management of pain and fatigue in the joint hypermobility syndrome (a.k.a. Ehlers-Danlos syndrome, hypermobility type): principles and proposal for a multidisciplinary approach. Am J Med Genet A 2012; 158A:2055-2070. 